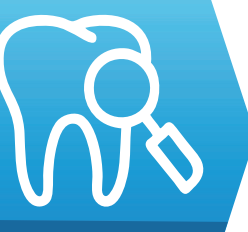

DENTAL MEDICINE

1) Prosthodontics Department, Iuliu Hatieganu University of Medicine and Pharmacy, Cluj-Napoca, Romania

2) Dental Propedeutics and Aesthetics Department, Iuliu Hatieganu University of Medicine and Pharmacy, Cluj-Napoca, Romania

3) Craniomaxillofacial Surgery Department, Iuliu Hatieganu University of Medicine and Pharmacy, Cluj-Napoca, Romania
DOI: 10.15386/cjmed-1131

Manuscript received: 06.08.2018

Received in revised form: 20.11.2018

Accepted: 26.11.2018

Address for correspondence:

bogdanculic@yahoo.com

\title{
A comparative study of the accuracy of five CAD-CAM systems
}

Smaranda Buduru ${ }^{1}$, Bogdan Culic ${ }^{2}$, Daniel Talmaceanu ${ }^{3}$, Adorjan Pal ${ }^{1}$

\begin{abstract}
Background and aims. The study aims at comparing the precision of different $\mathrm{CAD} /$ CAM systems: original systems built by the same manufacturer as opposed to systems whose components are built by different manufacturers.

Methods. We used the same physical cast which was scanned with five different scanners. The design of the PMMA copings was done by the same operator for the five virtual casts. Each coping was milled three times by each milling machine, thus obtaining 75 copings. The internal space was analyzed by scanning the replicas in light body silicone.
\end{abstract}

Results. The results analyzed the vertical marginal space (VMS) at the preparation limit, the axial internal space (AIS) and the internal occlusal space (IOS). For VMS the best results were obtained for scanner no 5 (S5) and milling machine 3 (M3). For AIS the best results were obtained with $\mathrm{S} 4$ and $\mathrm{M} 3$ combination. For IOS the best results were obtained with S45 and M3 combination.

Conclusions. According to the results of the study, we are able to confirm the working hypothesis by stating the following conclusions: the quality of the prosthetic elements obtained through $\mathrm{CAD} / \mathrm{CAM}$ processing differs depending on the CAD/CAM system used and is influenced by each component of every system individually, both the scanner and the milling unit; changing/switching any component of the CAD/CAM system has an impact on the quality of the resulting prosthetic work, but in an unpredictable manner.

Keywords: CAD/CAM, Monobrand/Hybrid systems, vertical marginal space

\section{Background and aims}

Amongthecutting-edgetechnologies available in dental medicine, CAD/CAM systems based on 3D scanners (intraoral or model), computer aided design (CAD) and computer aided manufacturing (CAM) in different (additive or subtractive) forms, have witnessed a remarkable development in the last years. The main target for using $\mathrm{CAD} / \mathrm{CAM}$ systems in dental care is related to the desire to eliminate human error in the manufacturing of prosthetic elements. The variety of $\mathrm{CAD} / \mathrm{CAM}$ systems available on the market raises several questions concerning the quality of prosthetic works manufactured by using different systems. The technological complexity, structural variety or IT diversity, namely the vast number of manufacturers, also account for the qualitative differences between prosthetic works manufactured by using different $\mathrm{CAD} / \mathrm{CAM}$ systems.

The distinctive variables of these systems make even more difficult the predictability and reproducibility of the results, thus leading to various and complex qualitative and functional discrepancies.

Although there are many studies [14] dedicated to various qualitative aspects specific of prosthetic works manufactured by using CAD/CAM systems, the conclusions of the papers are often contradictory and do not supply at first sight a clear picture of the predictability of the investigated systems or of the factors that have an impact on the quality of prosthetic works.

The study aims at comparing the precision of different CAD/CAM systems, original systems built by the same manufacturer, as well as systems whose components are built by different manufacturers. The working hypothesis is to establish the superiority of the hybrid (scanner and milling unit from different manufacturers) over the original monobrand system (scanner and milling unit originating from the same manufacturer) or vice versa. 


\section{Methods}

In the present study, we decided to make 75 PMMA copings by using 5 different lab scanners and 5 different milling units. We used a tooth (4.6) prepared for the substructure (the coping) of a crown. The 75 PMMA copings were made on the tooth by using 5 different scanners and they were then analyzed from different perspectives: vertical marginal space at the preparation limit, axial internal space at the middle of the axial wall height and internal occlusal space in 2 distinct points.

Five $\mathrm{CAD} / \mathrm{CAM}$ systems installed in different laboratories were used in the study, and they were selected according to the following inclusion criteria: milling unit with the same number of rotation axes, below $15 \mu \mathrm{m}$ acknowledged scanner precision (according to the technical data published by the manufacturer), model manufactured after 2014, PMMA milling capacity, open system or one that has modules allowing the users to work with open .STL files, compatibility with exocad dentalCAD 2016. In order to avoid potential ethical or legal issues, we decided to encrypt the commercial denomination of the CAD/CAM systems included in the study. The configuration of the systems based on the codes resulted after randomization is detailed in table I.

We used the letter "C" for complete systems consisting of a scanner (denoted by "S") and a milling unit (denoted by "M") provided by the same manufacturer. The table reveals that $\mathrm{C} 1=\mathrm{S} 1+\mathrm{M} 1, \mathrm{C} 2=\mathrm{S} 2+\mathrm{M} 2 \ldots . \mathrm{C} 5=\mathrm{S} 5+\mathrm{M} 5$ (i.e. $\mathrm{Cx}=\mathrm{Sx}+\mathrm{Mx}$ ). Twenty-five other $\mathrm{CAD} / \mathrm{CAM}$ systems with components from different manufacturers can be obtained by recombination (Sy+Mx).

A working cast with mobile abutment for tooth 4.6 (the homothetic tooth reduction was done with $1.2 \mathrm{~mm}$ chamfer margin and $1.5 \mathrm{~mm}$ occlusal surface reduction) was fabricated and mounted on the semi-adjustable articulator. The cast was sent to be scanned to 5 different laboratories that had their respective $5 \mathrm{CAD} / \mathrm{CAM}$ systems included in the study, thus producing 5 different virtual casts of the same physical working cast. All 5 virtual casts were then sent to the same design center separately in order to design the copings using the CAD. We decided to manufacture PMMA copings. One coping was designed for each virtual model, abiding by the same criteria and set parameters during the design stage: 0 vertical marginal space, $50 \mu \mathrm{m}$ cement gap, and coping thickness of $1 \mathrm{~mm}$ evenly following the contour of the preparation, which decreases towards the marginal area depending on the thickness required by the marginal design. The exocad DentalCAD 2016 software was used by the same operator, on the same computer simultaneously, and on the same day.

Thus, 5 virtual copings were designed and saved in .STL files, originating from the same physical cast and identically designed under the same circumstances; the only difference between the 5 copings was that the virtual cast came from different scanners. Each virtual coping was sent to all the 5 milling units, so each laboratory in part received all the 5 .STL files for milling. Consequently, each milling unit made 3 copies of all 5 virtual copings, that is 5 virtual copings $x 5$ milling units $\times 3$ copies $=75$ copings (Figure 1 ).

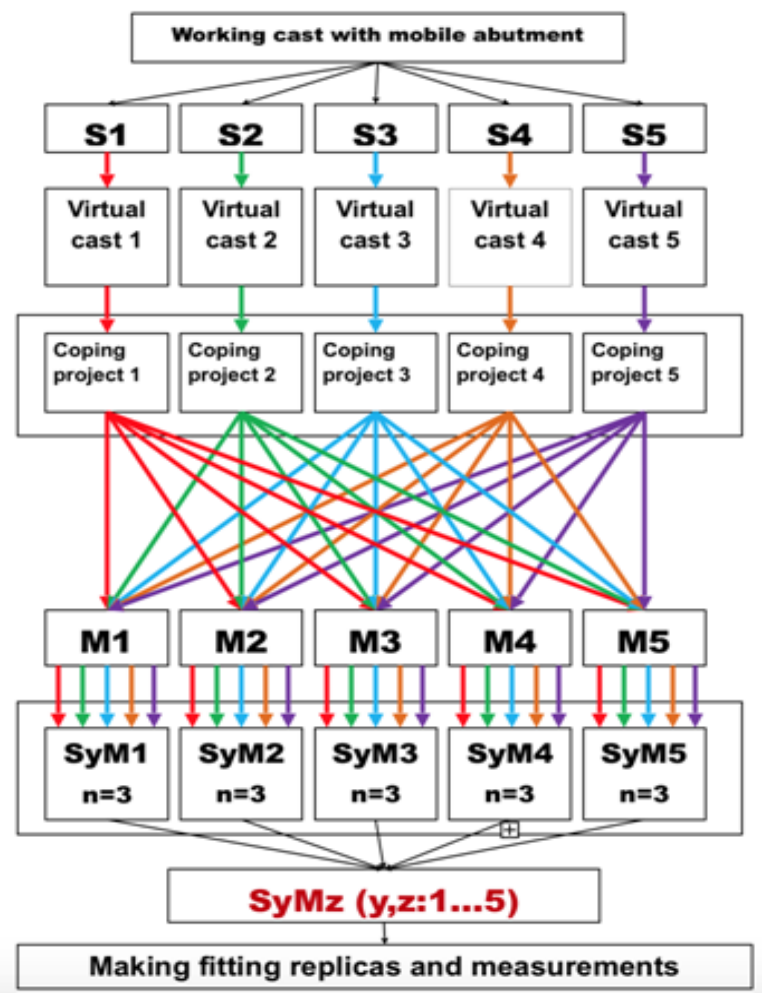

Figure 1. Schematic representation of the design process.

Standard 98 and 95ZZ Bilkim PMMA discs, $16 \mathrm{~mm}$ in width and A2 shade were used for milling.

Table I. Encryption of the CAD/CAM systems and components.

$\begin{array}{llllll}\mathbf{5} \text { monobrand systems } & \text { C1 } & \text { C2 } & \text { C3 } & \text { C4 } & \text { C5 } \\ \mathbf{5} \text { scanners } & \text { S1 } & \text { S2 } & \text { S3 } & \text { S4 } & \text { S5 } \\ \mathbf{5} \text { milling units } & \text { M1 } & \text { M2 } & \text { M3 } & \text { M4 } & \text { M5 } \\ & \text { S1M1 } & \text { S2M1 } & \text { S3M1 } & \text { S4M1 } & \text { S5M1 } \\ \mathbf{2 5} \text { new CAD/CAM systems (obtained by } & \text { S1M2 } & \text { S2M2 } & \text { S3M2 } & \text { S4M2 } & \text { S5M2 } \\ \text { recombining the scanners and milling units of the } & \text { S1M3 } & \text { S2M3 } & \text { S3M3 } & \text { S4M3 } & \text { S5M3 } \\ \text { monobrand systems) } & \text { S1M4 } & \text { S2M4 } & \text { S3M4 } & \text { S4M4 } & \text { S5M4 } \\ & \text { S1M5 } & \text { S2M5 } & \text { S3M5 } & \text { S4M5 } & \text { S5M5 }\end{array}$


In order to evaluate how the copings fit on the physical cast, a replica of the internal and marginal space was made using light body Polyvinyl Siloxane from a cartridge with two components, later fixed with putty Polyvinyl Siloxane. Materials used: a. cylinder to stabilize the mobile abutment, filled with putty Polyvinyl Siloxane $2 \mathrm{~mm}$ below the marginal preparation to hold the mobile abutment in central position; $b$. piston to place the coping on the abutment, to which a column-shaped container with 1.5 1 water is attached (the water maintains constant pressure); c. support for the water container meant to maintain a stable direction of the force the water exerts on the coping and on the abutment; $d$. lid for stabilizing the light body silicone into the putty silicone, which has 2 markings on the inside providing the reference points for precise cutting in the next stage. In practice, a set quantity of light body silicone was used to take an impression of the internal and marginal space for each coping, and then the piston with the water container was positioned perpendicularly on the occlusal surface of the coping so that the force were directly exerted on the central axis of the mobile abutment. The force was applied for 90 seconds, then the piston and the coping were removed and the thin layer of light body silicone remained attached to the mobile abutment. Next, the putty silicone was put in the application lid by using the same piston with the attached water container of 1.51 for 90 seconds. After removing the application lid, the fluid silicone was attached and stabilized into the putty silicone (Figure 2).

The silicone blocks were sectioned in buccallingual and mesial-distal direction on the cutting reference points marked on the inner side of the stabilizing lid. We used a professional, $120 \mathrm{~W}$ power, self-sharpening cutting tool with smooth edge disc and stabilizing system for the silicone block according to the markings (Figure 3).
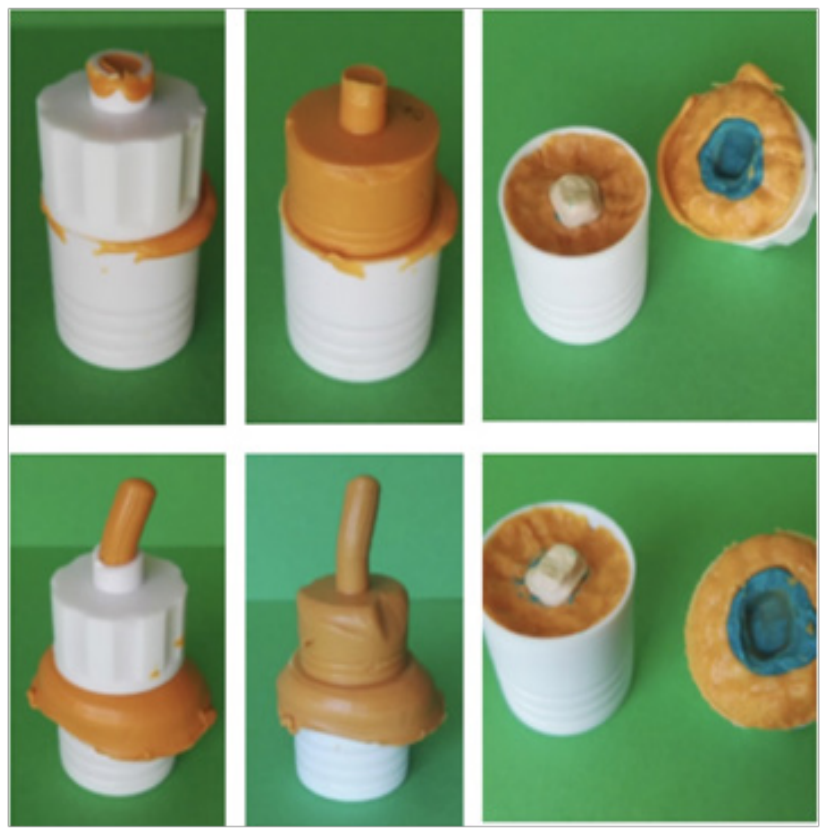

Figure 2. Internal space impression.
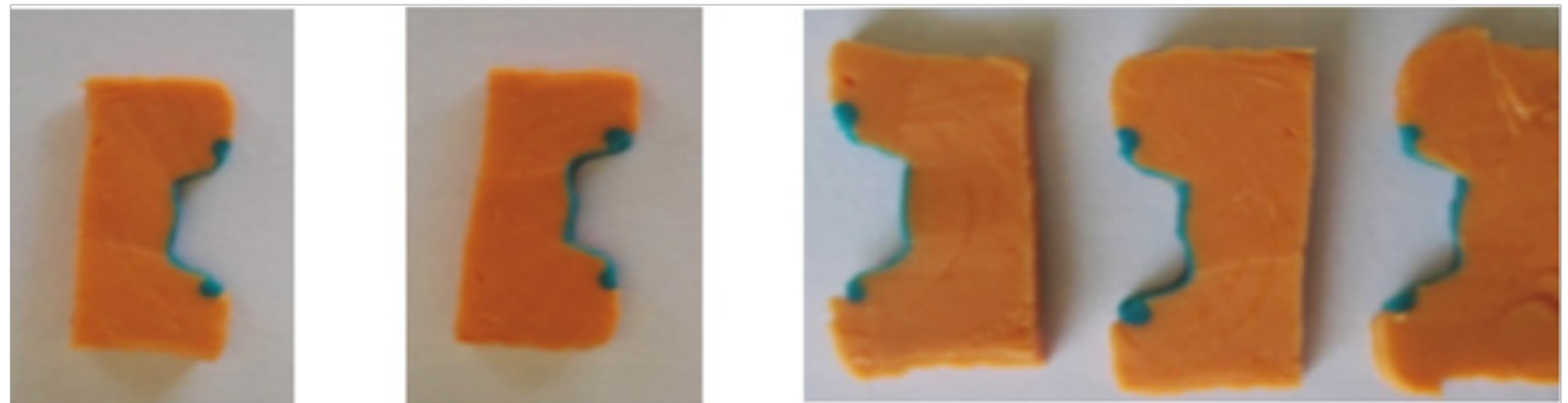

Figure 3. Sections of the internal replicas prepared for scanning. 
Each silicone block was cut into $2 \mathrm{~mm}$-thick slices, and each slice was scanned from the same direction using a Konica Minolta BizHub C452 scanner at a maximum resolution of $600 \mathrm{dpi}(7015 \times 4960$ pixels). The digital images thus acquired were imported into the Bersoft Image Management software program, which measures the distances between 2 points of the image in the analysis window using its own measurements units (data units) that are pixel fractions. In the Bersoft Image Management program, we determined the thickness of the light body silicone layer in 6 points for each section, as follows:

- Vertical marginal space at the preparation limit, both buccally and orally ( 2 measurements), using the abbreviations VMS (VMSb for the buccal area, and VMSo for the oral area);

- Axial internal space at the middle of the axial wall height, both buccally and orally; the measurement was carried out perpendicularly on the axial wall (2 measurements), using the abbreviations AIS (AISb and AISo);

- Internal occlusal space in 2 distinct points, the tip of the buccal cusps resulted after preparation and the central fossa, namely 2 measurements abbreviated IOSc and IOSf.

The resulting values were expressed in data units (fractions/multiples of pixels), converted then into $\mu \mathrm{m}$ with the conversion application.

\section{Results}

Based on the values obtained after converting the units measured for VMS, AIS, and IOS in $\mu \mathrm{m}$, we proceeded to applying the "Descriptive Statistics"/Data analysis from Microsoft Office Excel, which emphasized the following: the means, modules and medians had almost equal values, while the values of asymmetry (Skewness) and of the excess (Kurtosis) were close to 0, with some isolated exceptions. By analyzing these values, and by taking into account the rather small sample size under investigation, we concluded that the data recorded in the experimental study should be regarded as parametrical data with a relatively normal distribution, therefore we applied the Fisher test (the $f$ test) in order to perform variance analysis. The test resulted in equal or unequal variances, according to which we performed the corresponding test for each case individually. The results are summarized in tables II, III and IV.

Tables V, VI, and VII display the precision results for the tested systems.

Table II. "p" values after applying the $t$ test for the VMS means.

$\begin{array}{lcccccc} & \text { S1 } & \text { S2 } & \text { S3 } & \text { S4 } & \text { S5 } & \\ \text { M1 } & & 0.6461 & 0.6554 & 0.1239 & \mathbf{0 . 0 2 2 8} & \text { S1 } \\ \text { M2 } & 0.3220 & & 0.3559 & 0.3087 & 0.1081 & \text { S2 } \\ \text { M3 } & \mathbf{0 . 0 0 8 2} & \mathbf{0 . 0 0 7 9} & & \mathbf{0 . 0 0 9 2} & \mathbf{0 . 0 0 0 6} & \text { S3 } \\ \text { M4 } & \mathbf{0 . 0 4 7 2} & 0.0941 & \mathbf{0 . 0 2 7 3} & & 0.1631 & \text { S4 } \\ \text { M5 } & 0.7455 & 0.3714 & \mathbf{0 . 0 1 1 1} & 0.3766 & & \text { S5 } \\ & \text { M1 } & \text { M2 } & \text { M3 } & \text { M4 } & \text { M5 } & \end{array}$

Table III. "p" values after applying the $t$ test for the AIS means.

$\begin{array}{lcccccc} & \text { S1 } & \text { S2 } & \text { S3 } & \text { S4 } & \text { S5 } & \\ \text { M1 } & & 0.8299 & 0.1046 & \mathbf{0 . 0 3 8 6} & \mathbf{0 . 0 3 9 4} & \text { S1 } \\ \text { M2 } & 0.6039 & & 0.4019 & 0.1164 & 0.1182 & \text { S2 } \\ \text { M3 } & 0.0608 & 0.1770 & & \mathbf{0 . 0 0 0 7} & \mathbf{0 . 0 0 7 3} & \text { S3 } \\ \text { M4 } & \mathbf{0 . 0 3 0 1} & 0.2957 & 0.5084 & & 0.9889 & \text { S4 } \\ \text { M5 } & 0.2260 & 0.6021 & 0.3240 & 0.5923 & & \text { S5 } \\ & \text { M1 } & \text { M2 } & \text { M3 } & \text { M4 } & \text { M5 } & \end{array}$

Table IV. "p" values after applying the $t$ test for the IOS means.

$\begin{array}{ccccccc} & \text { S1 } & \text { S2 } & \text { S3 } & \text { S4 } & \text { S5 } & \\ \text { M1 } & & 0.9492 & 0.2739 & \mathbf{0 . 0 2 7 9} & \mathbf{0 . 0 2 1 7} & \text { S1 } \\ \text { M2 } & 0.8786 & & 0.5447 & \mathbf{0 . 0 4 9 9} & 0.5779 & \text { S2 } \\ \text { M3 } & \mathbf{0 . 0 4 9 7} & 0.4492 & & \mathbf{0 . 0 0 4 2} & \mathbf{0 . 0 0 0 1} & \text { S3 } \\ \text { M4 } & \mathbf{0 . 0 0 1 7} & 0.3732 & 0.9971 & & 0.8849 & \text { S4 } \\ \text { M5 } & 0.7197 & 0.6763 & 0.5177 & 0.7174 & & \text { S5 } \\ & \text { M1 } & \text { M2 } & \text { M3 } & \text { M4 } & \text { M5 } & \end{array}$


Table V. Precision classification of the tested components and systems.

\begin{tabular}{|c|c|c|c|c|c|c|c|c|}
\hline Space & \multicolumn{5}{|c|}{ Components classification } & \multicolumn{3}{|c|}{ Top 3 systems } \\
\hline & I & II & III & IV & $\mathbf{V}$ & I & II & III \\
\hline \multirow{3}{*}{ VMS } & $\mathrm{S} 3$ & S1 & S2 & S4 & S5 & \multirow{3}{*}{ S3M2 } & \multirow{3}{*}{$\mathrm{S} 2 \mathrm{M} 2$} & \multirow{3}{*}{ S2M1 } \\
\hline & M2 & M1 & M5 & M4 & M3 & & & \\
\hline & C2 & C1 & C5 & $\mathbf{C 3}$ & $\mathrm{C4}$ & & & \\
\hline \multirow{3}{*}{ AIS } & S3 & S2 & S1 & S4 & S5 & \multirow{3}{*}{$\mathrm{S} 2 \mathrm{M} 1$} & \multirow{3}{*}{ S3M5 } & \multirow{3}{*}{ S3M1 } \\
\hline & M1 & M2 & M5 & M4 & M3 & & & \\
\hline & C3 & $\mathrm{C2}$ & C1 & C5 & $\mathrm{C} 4$ & & & \\
\hline \multirow{3}{*}{ IOS } & S3 & S1 & S2 & S4 & S5 & \multirow{3}{*}{ S1M1 } & \multirow{3}{*}{ S3M3 } & \multirow{3}{*}{$\mathrm{S} 2 \mathrm{M}$} \\
\hline & M1 & M2 & M3 & M5 & M4 & & & \\
\hline & C1 & C3 & $\mathrm{C} 2$ & $\mathrm{C} 4$ & C5 & & & \\
\hline
\end{tabular}

Table VI. Precision of monobrand systems.

\begin{tabular}{|c|c|c|c|c|c|}
\hline SxMx=Cx & S1M1=C1 & S2M2=C2 & S3M3=C3 & S4M4=C4 & S5M5=C5 \\
\hline VMS & 45.83 & 28.83 & 79.83 & 94.00 & 75.00 \\
AIS & 97.50 & 62.50 & 62.33 & 161.33 & 149.83 \\
IOS & 70.50 & 141.50 & 82.50 & 199.50 & 207.83 \\
\hline
\end{tabular}

Table VII. Classification of monobrand systems.

\begin{tabular}{lcccccc}
\multicolumn{7}{c}{ Based exclusively on scanning precision } \\
& 1 & 2 & 3 & 4 & 5 \\
VMS & C1 & C2 & C5 & C3 $=$ C4 \\
AIS & \multicolumn{1}{c}{ C3 $=$ C2 } & C1 & C5 & C4 \\
IOS & C1 & C3 & C2 & \multicolumn{2}{c}{ C4 $4=$ C5 }
\end{tabular}

\section{Discussion}

Even if all 5 scanners operate according to the same optical principle and use blue light, the built-in optical cameras differ in terms of resolution and focalizing speed. The inspection of the milled copings did not reveal any important differences in terms of surface finishing, milling quality or marginal area integrity. Therefore, we can assert that all the tested systems displayed similar performances in terms of qualitative surface and marginal integrity parameters. In the first fitting on the abutment, without light body silicone, all the copings showed good, similar, adequate marginal adaptation, being seated on the marginal preparation without any distancing or visually detectable VMS oscillations.

The inspection of the internal and marginal space light body silicone replicas of all the copings on the abutment immediately after their removal endorsed the conclusion of other studies, namely that the internal space is not uniform, as it should ideally be according to the CAD design. There were several more transparent silicone zones (areas), with thinner silicone layers, and the transparent zones/areas were not in the same region at each impression- taking, but differed from one impression to another or from one coping to the other.

The obtained values of the VMS, AIS and IOS showed that the CAD/CAM systems did not conform to the CAD settings and did not faithfully reproduce the space designed and intended for the cement gap. Therefore, VMS was never 0 , and the measured IOS was not equal to the measured VMS added to the CAD spacing. We noticed that in the case of the 3 theoretically identical copings, milled by each system apart, the VMS, AIS or IOS were not identical either, or all measured values were the same. Even if the differences between the 3 "identical" copings were usually minor and statistically insignificant, they, however, should not have existed in theory.

Following the analysis of individual results, the best values were generally found in the new combinations of components built by different manufacturers.

The study enabled us to check the statistical significance of the differences between each system apart and all the other analyzed systems and the result was that from 300 possible combinations (combinations of 25 taken 2 at a time) only 59 proved statistically insignificant, i.e. 
$19 \%$. In other words, $81 \%$ of the differences revealed by the study between the precision of the tested systems were statistically significant; consequently in $81 \%$ of the cases 1 of the 2 compared systems displayed a significantly higher precision than the other. The gold standard for quality evaluation and clinical success of fixed prosthetic is marginal adaptation [1,2]. Usually, the VMS is set to 0 beginning with the CAD stage, and the internal space, depending on manifold factors, may be set to different values ranging between $20-80 \mu \mathrm{m}[3,4]$.

A series of studies have established the optimal range for the physical VMS of prosthetics between 40 and $120 \mu \mathrm{m}[5,6,7]$. Other studies indicate that the maximum acceptable limit is $100 \mu \mathrm{m}$; consequently, it can be stated that the generally acknowledged clinically acceptable range for the marginal space is $40-100 \mu \mathrm{m}$ [8]. The literature in the field indicates different values for the VMS [9]: for zirconia-based dental prosthetics, values ranging between 60 and $74 \mu \mathrm{m}$ are mentioned [9,10]; for ceramic single unit fixed partial dentures, $64-83 \mu \mathrm{m}$ [10], $50-75 \mu \mathrm{m}$ [11], a mean of $56 \mu \mathrm{m}$ [12] or $51.7 \mu \mathrm{m}$ [13]. Other studies range the VMS between 39.1 and $201 \mu \mathrm{m}$ or 10 and $110 \mu \mathrm{m}$. Some sources indicate that the VMS values range between 9.94 and $308.92 \mu \mathrm{m}$ [14]. The AIS value ranges also vary, but on a larger scale: $23-230 \mu \mathrm{m}, 23.5-219.12 \mu \mathrm{m}, 51-$ $442 \mu \mathrm{m}$ [15]. Most of the studies indicate VMSs below 80 $\mu \mathrm{m}$, a value considered to be clinically acceptable, however far above the ADA recommended range for the VMS (25 $40 \mu \mathrm{m})$ [15].

The results should be interpreted taking into account the limitations of the study both in what concerns the investigated $\mathrm{CAD} / \mathrm{CAM}$ systems (located in different environments, operated by different specialists, with different burs, some of the settings, use time or wear, etc.) and the measurements and determinations (difference in the rheological properties of the silicone and of the cementation materials, different application load in contrast with the clinically applied force when cementing, elastic properties of the silicone not present in cements, the possibility to compress the light body silicone layer during stabilization with putty silicone, etc.). In relation to the methods of control of the investigated variables mentioned by the surveyed literature in the field, the present study fits into the common standards of studying the CAD/CAM precision in vitro. Another important limitation arises from the number of milled copings for each individual case - in our study the number is $n=3$ - as well as from the number of sections, namely number of measurements performed for each section.

\section{Conclusions}

According to the results of the study and taking into account the given limitations, we are able to confirm the working hypothesis by stating the following conclusions: the quality of prosthetic elements obtained through
$\mathrm{CAD} / \mathrm{CAM}$ processing differs depending on the $\mathrm{CAD} /$ CAM system used and is influenced by each component of every system apart, both the scanner and the milling unit; changing/switching any component of the CAD/ CAM system has an impact on the quality of the resulting prosthetic work, but in an unpredictable manner.

The internal space of the prosthetic work built with the CAD/CAM systems is not uniform and displays important variations depending on the surface and height at which the measurement is taken. The spacing value set during the CAD stage (the thickness of the space designed for the cementing material) always influences the final VMS, sometimes inversely proportional and unpredictably, and the exact initially set value of the spacing is not preserved in the internal space of the milled prosthetic piece. We may configure new CAD/CAM systems using CAD/CAM components built by different manufacturers. These new systems may ensure a superior quality of the finite product over the original systems.

\section{References}

1. Beuer F, Schweiger J, Edelhoff D. Digital dentistry: an overview of recent developments for CAD/CAM generated restorations. $\mathrm{Br}$ Dent J. 2008;204:505-511.

2. Wittneben JG, Wright RF, Weber HP, Gallucci GO. A systematic review of the clinical performance of $\mathrm{CAD} / \mathrm{CAM}$ single-tooth restorations. Int J Prosthodont. 2009;22:466-471.

3. Martínez-Rus F, Suárez MJ, Rivera B, Pradíes G. Influence of $\mathrm{CAD} / \mathrm{CAM}$ systems and cement selection on marginal discrepancy of zirconia-based ceramic crowns. Am J Dent. 2012;25:67-72.

4. Boitelle P, Mawussi B, Tapie L, Fromentin O. A systematic review of CAD/CAM fit restoration evaluations. J Oral Rehabil. 2014;41:853-874.

5. Vojdani M, Safari A, Mohaghegh M, Pardis S, Mahdavi F. The effect of porcelain firing and type of finish line on the marginal fit of zirconia copings. J Dent (Shiraz). 2015;16:113-120.

6. Coli P, Karlsson S. Precision of a CAD/CAM technique for the production of zirconium dioxide copings. Int J Prosthodont. 2004; 17:577-580.

7. Tinschert J, Natt G, Mautsch W, Spiekermann H, Anusavice KJ. Marginal fit of alumina-and zirconia-based fixed partial dentures produced by a CAD/CAM system. Oper Dent. 2001;26:367-374. 8. Hung SH, Hung KS, Eick JD, Chappell RP. Marginal fit of porcelain-fused-to-metal and two types of ceramic crown. J Prosthet Dent. 1990;63:26-31.

9. Reich S, Wichmann M, Nkenke E, Proeschel P. Clinical fit of all-ceramic three-unit fixed partial dentures, generated with three different CAD/CAM systems. Eur J Oral Sci. 2005;113:174-179.

10. McLaren E. CAD/CAM Dental Technology: A Perspective on Its Evolution and Status. Compendium of Continuing Education in Dentistry. 2011, vol.32, iss. Available from: https:// www.aegisdentalnetwork.com/cced/2011/05/cad-cam-dentaltechnology-a-perspective-on-its-evolution-and-status

11. Aeran H, Kumar V, Seth J, Sharma A. Computer aided designing-computer aided milling in prosthodontics: A promising technology for future. IJSS Case Reports \& Reviews.2014;1:23-27. 12. Gonzalo E, Suárez MJ, Serrano B, Lozano JF. Comparative analysis of two measurement methods for marginal fit in 


\section{Original Research}

metal-ceramic and zirconia posterior FPDs. Int J Prosthodont. 2009;22:374-377.

13. Nawafleh NA, Mack F, Evans J, Mackay J, Hatamleh MM. Accuracy and reliability of methods to measure marginal adaptation of crowns and FDPs: a literature review. J Prosthodont. 2013;22:419-428.
14. Kale E, Seker E, Yilmaz B, Özcelik TB. Effect of cement space on the marginal fit of CAD-CAM-fabricated monolithic zirconia crowns. J Prosthet Dent. 2016;116:890-895.

15. Kokubo Y, Tsumita M, Kano T, Sakurai S, Fukushima S. Clinical marginal and internal gaps of zirconia all-ceramic crowns. J Prosthodont Res. 2011;55:40-43. 